\title{
Bacterial Outer Membrane Vesicles from Dextran Sulfate Sodium-Induced Colitis Differentially Regulate Intestinal UDP-Glucuronosyltransferase 1A1 Partially Through Toll-Like Receptor 4/Mitogen-Activated Protein Kinase/Phosphatidylinositol 3-Kinase Pathway ${ }^{\mathbb{S}}$
}

\author{
Xue-Jiao Gao, Ting Li, Bin Wei, Zhi-Xiang Yan, Nan Hu, Yan-Juan Huang, Bei-Lei Han, \\ Tai-Seng Wai, Wei Yang, and Ru Yan
}

State Key Laboratory of Quality Research in Chinese Medicine, Institute of Chinese Medical Sciences, University of Macau, Macao, China; and Zhuhai UM Science \& Technology Research Institute, Zhuhai, China

Received October 24, 2017; accepted January 3, 2018

\section{ABSTRACT}

UDP-glucuronosyltransferase 1A1 (UGT1A1) constitutes an important part of intestinal epithelial barrier and catalyzes glucuronidation of many endogenous compounds and drugs. Downregulation of UGT1A1 in inflammation has been reported, whereas the association with gut dysbiosis is poorly defined. This study verified the involvement of gut microbiota in intestinal UGT1A1 regulation using dextran sulfate sodium (DSS)-induced rat colitis model plus fecal microbiota transplantation (FMT). Generally, both DSS induction and colitis-to-normal FMT suppressed mRNA and protein expressions of UGT1A1 and nuclear xenobiotic receptors (NRs) in colon, but enhanced mRNA and decreased protein of rat UGT1A1/rat NRs in small intestine. Normal-to-colitis FMT alleviated DSS-induced changes. Bacterial outer membrane vesicles (OMVs) from colitis rats and rats receiving colitis feces reduced both $\mathrm{WRNA}$ and protein of human UGT1A1 (hUGT1A1)/human NRs (hNRs) in Caco-2 cells. Interestingly, using deoxycholate to reduce lipopolysaccharide, normal OMVs upregulated hUGT1A1/hNRs, whereas colitis OMVs decreased, indicating the involvement of other OMVs components in UGT1A1 regulation. The $10-$ to $50-\mathrm{kDa}$ fractions from both normal and colitis OMVs downregulated hUGT1A1, human PXR, and human PPAR- $\gamma$, whereas $>50-k D a$ fractions from normal rats upregulated hUGT1A1 and human CAR. Additionally, the conditioned medium from OMVs-stimulated rat primary macrophages also reduced hUGT1A1/hNRs expression. Both Toll-like receptor (TLR)2 and TLR4 were activated by DSS, colitis-to-normal FMT, and the opposite, whereas only TLR4 was increased in OMVs-treated cells. TLR4 small interfering RNA blocked hUGT1A1/hNRs downregulation and phosphatidylinositol 3-kinase/Akt, extracellular signal-regulated kinase, and nuclear factor $\kappa$ B phosphorylation evoked by bacterial OMVs. Taken together, this study demonstrated that gut microbiota regulate intestinal UGT1A1 partially through secreting OMVs, which interact with intestinal epithelial cells directly or via activating macrophage.
Introduction

UDP-glucuronosyltransferase 1A1 (UGT1A1), one of the major intestinal drug-metabolizing enzymes, catalyzes glucuronidation of many potentially harmful compounds and drugs. Inhibition of UGT1A1

This work was supported by the National Natural Science Foundation [81473281], the Science and Technology Development Fund of Macao SAR [043/2011/A2, 029/2015/A1], and University of Macau [MYRG2015-00220-ICMSQRCM].

https://doi.org/10.1124/dmd.117.079046.

$\$$ This article has supplemental material available at dmd.aspetjournals.org. may bring increased risks of drug-drug interactions and cause bilirubinrelated diseases (Strauss et al., 2006). Existing data indicate an involvement of gut microbiota in regulating UGT1A1 or its upstream regulators, the nuclear xenobiotic receptors (NRs) pregnane $\mathrm{X}$ receptor (PXR), constitutive androstane/active receptor (CAR), and peroxisome proliferator activation receptors (PPARs). Exposure to lipopolysaccharide (LPS) or Citrobacter rodentium infection downregulated hepatic UGT1A1 in mice (Richardson et al., 2006). Indole 3-propionic acid produced by commensal Clostridium sporogenes promoted PXR mRNA expression in $\mathrm{Nr}_{1} 2^{-/-} \mathrm{Tlr} 4^{-/-}$mice (Venkatesh et al., 2014). However, the contribution of gut microbiota community as a whole to

ABBREVIATIONS: BCA, bicinchoninic acid; CAR, constitutive and rostane/active receptor; CM, conditioned medium; DAI, disease activity index; DMEM, Dulbecco's modified Eagle's medium; DOC, deoxycholate; DSS, dextran sulfate sodium; ELISA, enzyme-linked immunosorbent assay; ERK, extracellular signal-regulated kinase; FMT, fecal microbiota transplantation; GAPDH, glyceraldehyde-3-phosphate dehydrogenase; hCAR, human CAR; hNR, human NR; hPPAR, human PPAR; hPXR, human PXR; hUGT1A1, human UGT1A1; IECs, intestinal epithelial cells; IFN, interferon; IL, interleukin; LPS, lipopolysaccharide; MAPK, mitogen-activated protein kinase; MCP, monocyte chemoattractant protein; MPO, myeloperoxidase; NC, negative control; NF- $\kappa$ B, nuclear factor $\kappa \mathrm{B}$; NN, normal-to-normal FMT; NR, nuclear xenobiotic receptor; NU, colitis-to-normal FMT; OMVs, bacterial outer membrane vesicles; PBS, phosphate-buffered solution; PCR, polymerase chain reaction; PI3K, phosphatidylinositol 3-kinase; PPAR, peroxisome proliferator activation receptor; PVDF, polyvinylidene difluoride; PXR, pregnane X receptor; rCAR, rat CAR; rNR, rat NR; rPPAR, rat PPAR; rPXR, rat PXR; RT, reverse transcription; rUGT1A1, rat UGT1A1; siRNA, small interfering RNA; TGF, transforming growth factor; TLR, Tolllike receptor; TNF, tumor necrosis factor; UC, ulcerative colitis; UGT1A1, UDP-glucuronosyltransferase 1A1; UN, ; IECs, intestinal epithelial cells. 
UGT1A1 regulation in intestinal epithelial cells (IECs) remains to be addressed.

Ulcerative colitis (UC), a form of inflammatory bowel disease, is characterized with chronic local inflammatory responses and microbial imbalance. Commensal bacteria or their products are drivers of dysregulated immunity in inflammatory bowel disease. Alterations of Gram-negative bacteria dominated gut dysbiosis in UC (Vigsnæs et al., 2012). Gram-negative bacteria interact with the host by producing secretory nano-sized outer membrane vesicles (OMVs) to deliver cohort of soluble and insoluble components into host cells (Ellis and Kuehn, 2010). LPS, one of the most abundant components of OMVs, is considered as the primary initiator for the pathogenic activities of OMVs (Beutler and Rietschel, 2003). LPS has been demonstrated to be involved in regulation of drug-metabolizing enzymes and transporters (Lu et al., 2008; Morgan et al., 2008). OMVs mediated the cytosolic localization of LPS (Vanaja et al., 2016). OMVs also contain other components (proteins, peptidoglycan, DNA, RNA, etc.) that can elicit inflammatory responses (Kaparakis-Liaskos and Ferrero, 2015). Neisseria meningitidis OMVs showed more potent proinflammatory properties than LPS in human whole blood (Mirlashari et al., 2001). Thus, OMVs is more suitable than LPS as the materials to investigate Gramnegative bacteria-host interactions.

Diminished drug metabolic capability is associated with inflammation (Christensen and Hermann, 2012). Recent advances indicate a key role for innate immunity in colonic inflammation (Marks and Segal, 2008). A large population of macrophages inhabits in intestine and steer innate immune response. Contacting with invading microorganisms leads to polarization of macrophages to M1 type (classically activated) or M2 type (alternatively activated), causing intestinal tissue damage, or maintains intestinal homeostasis by secreting pro- and anti-inflammatory cytokines, respectively (Nakata et al., 2013). A significant increase of M2 macrophages has been observed in UC patients (Cosin-Roger et al., 2013). In colitis animals, dextran sulfate sodium (DSS) drives the macrophage phenotype toward the M2 lineage (Kono et al., 2016). Macrophage colony-stimulating factor-deficient (op/op) mice, which are not able to develop mature macrophages, show decreased susceptibility to DSS-induced colitis (Ghia et al., 2008). Moreover, OMVs from $N$. meningitidis could promote macrophage polarization (Tavano et al., 2009). Macrophages sensing both LPS and protein components of Pseudomonas aeruginosa OMVs contribute to bacterial strain-specific inflammatory responses (Ellis et al., 2010). To our best knowledge, there is no report linking macrophage polarization with intestinal UGT1A1 regulation.

Toll-like receptors (TLRs) are key participants in innate immune responses. Among 11 human TLRs, TLR2 and TLR4 could recognize structurally diverse molecules from microbe. Bacterial OMVs could elicit biologic effects and inflammatory responses through activating TLR2 and/or TLR4. P. aeruginosa OMVs induced lung inflammation partly through activating both TLR2 and TLR4 (Park et al., 2013). Escherichia coli OMVs upregulated cell adhesion molecules in human microvascular endothelial cells via nuclear factor $\kappa \mathrm{B}(\mathrm{NF}-\kappa \mathrm{B})$ and TLR4-dependent pathways (Kim et al., 2013).

Fecal microbiota transplantation (FMT) is arising as a promising therapeutic strategy for some gut dysbiosis-related diseases through transplanting healthy fecal bacteria into the gut lumen of a patient. It has been demonstrated successful in colitis and Clostridium difficile infection (Borody et al., 2013; van Nood et al., 2013). Transplantation of fecal microbiota, specific bacterial strains, or combinations is widely adopted in basic research to assess their roles in disease etiology, gene regulation, and drug interventions (Li et al., 2015).

In this study, the involvement of gut microbiota in intestinal UGT1A1 regulation was first examined by measuring intestinal UGT1A1 and major NRs in DSS-induced experimental colitis rat model and rats receiving FMT. The discriminative alterations of hUGT1A1 and human NRs (hNRs) in Caco-2 cells treated by OMVs (complete, different molecular-weight fractions, LPS-reducing) from normal and colitis rats, or conditioned medium from OMVs-stimulated macrophages, were characterized to delineate the contributions of OMVs and the role of macrophage polarization. At last, the involvement of TLR4/mitogenactivated protein kinase/phosphatidylinositol 3-kinase (PI3K) was assessed using TLR4 inhibition or TLR4 small interfering RNA (siRNA) transfection in Caco-2 cells.

\section{Materials and Methods}

Reagents. DSS (mol. wt.: 36,000-50,000) was purchased from MP Biomedicals (Santa Cruz, CA) and dissolved in deionized water to make a solution of $5 \%$ $(w / v)$. Enzyme-linked immunosorbent assay (ELISA) kits for measuring rat tumor necrosis factor (TNF)- $\alpha$, interleukin (IL)- $1 \beta$, IL-6, IL-4, IL-10, interferon (IFN)- $\gamma$, transforming growth factor (TGF)- $\beta 1$, and monocyte chemoattractant protein (MCP)-1 were purchased from Excell Biologic (Shanghai, China). The bicinchoninic acid (BCA) assay kit, Lipofectamine 2000 reagent, reverse-transcription (RT) kit, Pierce limulus amebocyte lysate chromogenic endotoxin quantitation kit, TRIzol, and sodium deoxycholate detergent were supplied by Thermo Fisher Scientific (Waltham, MA). SYBR Premix Ex Taq (Perfect Real-Time) polymerase chain reaction (PCR) kit and primers were obtained from TaKaRa (Guangzhou, China). TLR4 siRNA was purchased from GenePharma (Shanghai, China). Anti-phospho-Akt (Ser473) antibody (9271), phospho-NF- $\kappa$ B p65 (Ser536) antibody (3031), and horseradish peroxidase-conjugated anti-rabbit and anti-mouse IgG antibodies were supplied by Cell Signaling Technology (Shanghai, China). Enhanced chemiluminescence Plus Western blotting detection reagent was purchased from Beyotime Institute of Biotechnology (Nanjing, China). Antibodies for PXR (ab192579), PPAR- $\gamma$ (ab209350), CAR (ab62590), UGT1A1 (ab194697), TLR2 (ab108998), and TLR4 (ab22048) were purchased from Abcam (Cambridge, UK), and those for phosphorylated extracellular signalregulated kinase (ERK) 1/2 (sc-136521) and phosphorylated PI3K (Tyr467) (sc293115) were obtained from Santa Cruz Biotechnology (Santa Cruz, CA). Polyvinylidene difluoride (PVDF) and ultrafiltration membranes were purchased from Merck Millipore (Billerica, MA). TAK-242 (resatorvid) was purchased from ApexBio Technology (Houston, TX). Dulbecco's modified Eagle's medium (DMEM), fetal bovine serum, phosphate-buffered solution (PBS), and nonessential amino acids were products of Gibco (Waltham, MA).

Animals. Male Sprague-Dawley rats (250-300 g, 8 weeks) were provided by the Experimental Animal Facility of University of Macau (Macao, China) and housed in a temperature $\left(24^{\circ} \mathrm{C}\right)$ and humidity $(45 \%-55 \%)$-controlled room with a 12-hour light/dark cycle in a specific pathogen-free-class laboratory. Rats were placed in a conditioning chamber and allowed to acclimate to the new environment for 4 days prior to experiments with access to standard chow and reverse osmosis water ad libitum. The chow was comprised of corn, fish meal, wheat flour, salt, vitamins, trace elements, amino acids, etc. The care and treatment of the rats followed a protocol (UMAEC-2015-09) approved by the Animal Ethics Committee, University of Macau.

In Vivo Protocol. Rats were divided randomly into five groups (six animals each). Rats in Colitis group received 5\% DSS in drinking water for consecutive 7 days (day 0-day 7). Rats receiving drinking water alone served as controls (normal group). The fecal samples from normal and Colitis groups were freshly collected daily at 10:00 AM, and portions were pooled at equal amount within group. One gram pooled fecal samples was suspended in $10 \mathrm{ml}$ sterile $0.9 \%$ normal saline by vortexing, and then fecal suspensions were orally administered to each rat of $\mathrm{NN}$ (normal rats received normal feces) and $\mathrm{NU}$ (normal rats received Colitis feces) groups, respectively, at $1 \mathrm{~g} / \mathrm{kg}$ by gavage daily for 7 days. Rats in UN group received 5\% DSS in drinking water as well as oral administration of $1 \mathrm{~g} / \mathrm{kg}$ fecal suspension from normal group by gavage for 7 days. Throughout the experimental period, rats were fed standard chew and bottles were refilled daily with fresh DSS solution (Colitis and UN groups) or reverse osmosis water (normal, NN and NU groups). The body weight and stool consistency of each rat from all groups were recorded on a daily basis. Blood samples (200 $\mu \mathrm{l}$ each) were collected from orbital sinus on days $0,3,5$, and 7 . On the last day of the experiment (day 7), rats were sacrificed by cervical dislocation. 
Small intestines and colons were collected, flushed with ice-cold phosphatebuffered saline to move food particles, and then cut longitudinally into several segments. Intestinal and colonic mucosa was scraped from the smooth muscle using a glass microscope slide.

Assessment of Clinical Signs of Colitis. Disease activity index (DAI), histologic evaluation, cytokine determination, and myeloperoxidase (MPO) assay were carried out, as described previously (Huang et al., 2015), with minor modifications. Each rat was given a DAI score for weight loss, stool consistency, and bloody stool. Colon segment $(0.5 \mathrm{~cm})$ from the distal end of the colon was removed, and $4-\mu \mathrm{m}$ sections were obtained, stained with $\mathrm{H} \& \mathrm{E}$ to assess epithelial damage, architectural derangements, goblet cell depletion, edema/ulceration, and inflammatory cell infiltrate using Olympus CX21 microscope and an Olympus SC100 camera. Serum levels of IL-1 $\beta$, IL-4, IL-6, IL-10, and TNF- $\alpha$ were assayed using ELISA kits, according to manufacturer's instruction. For MPO assay, colonic mucosa was homogenized in $50 \mathrm{mM}$ phosphate buffer $(\mathrm{pH} 6.0$ ) containing $0.5 \%$ Hexadecyl-trimethylammonium bromide. The supernatant (5 $\mu \mathrm{l}$ ) was added to $20 \mu \mathrm{l}$ $2.5 \mathrm{mM}$ o-dianisidine hydrochloride and $75 \mu 10.00065 \% \mathrm{H}_{2} \mathrm{O}_{2}$ and kept for 5 minutes to record absorbance at $470 \mathrm{~nm}$ on Spectra Max M5 Multi-Mode Microplate Readers. Protein concentration was determined by BCA assay. One unit of MPO activity was defined as the amount of enzyme degrading $1 \mathrm{nM} \mathrm{H}_{2} \mathrm{O}_{2}$ per minute at $25^{\circ} \mathrm{C}$.

Preparation of Complete OMVs, Fractions, and Low-LPS OMVs. Fecal samples freshly collected on day 7 were pooled within group and homogenized (10\%, $\mathrm{w} / \mathrm{v}$ ) in $0.1 \mathrm{M}$ PBS, followed by centrifugation at $200 \mathrm{~g}$ for 10 minutes. The supernatant was collected and centrifuged at $4500 \mathrm{~g}$ for 30 minutes. Microbial OMVs were prepared as described previously (Eddy et al., 2014). The resultant precipitate was resuspended with Luria-Bertani broth to obtain gut microbiota suspension at $2 \mathrm{mg} / \mathrm{ml}$ and incubated at $37^{\circ} \mathrm{C}$ for 24 hours. Bacterial cultures were pelleted at $6000 \mathrm{~g}$ for 20 minutes. The supernatant was filtered through a $0.45-\mu \mathrm{m}$ vacuum filter, and the filtrate was further filtered through a $0.22-\mu \mathrm{m}$ vacuum filter to remove any remaining cells and then ultracentrifuged at $200,000 \mathrm{~g}$ for 4 hours at $4^{\circ} \mathrm{C}$. The pellets (OMVs) were suspended in PBS for experiments. The protein content was determined by BCA assay.

OMV preparations $(50 \mu \mathrm{g} / \mathrm{ml}, 10 \mathrm{ml})$ were further fractionated by ultrafiltration with 3-, 10-, 30-, and 50-kDa ultrafiltration membranes according to the manufacturer's protocol to obtain fractions as follows: $<3$ (F $<3), 3-10$ (F3-10), 10-30 (F10-30), 30-50 (F30-50), and $>50 \mathrm{kDa}(\mathrm{F}>50)$. Each fraction obtained was made up to $10 \mathrm{ml}$ to maintain the same proportion in the OMVs.

Low-LPS OMVs were prepared according to previous reports (Claassen et al., 1996; Zariri et al., 2016) with minor modifications. Briefly, the bacterial cultures were pelleted as described above and resuspended in $0.1 \mathrm{M}$ Tris-10 mM EDTA buffer. After incubation for 5 minutes at $4^{\circ} \mathrm{C}$, deoxycholate (DOC; $100 \mathrm{~g} / \mathrm{l}$ ) was added to reduce LPS from OMVs (DOC:Tris-EDTA, 1:20, v/v) and then ultracentrifuged at $200,000 \mathrm{~g}$ for 4 hours at $4^{\circ} \mathrm{C}$. The pellet was resuspended in $0.1 \mathrm{M}$ Tris- $10 \mathrm{mM}$ EDTA buffer containing $5 \mathrm{~g} / \mathrm{l}$ DOC and then filtered through a $0.22-\mu \mathrm{m}$ vacuum filter. The supernatant was then ultracentrifuged at $200,000 \mathrm{~g}$ for 4 hours at $4{ }^{\circ} \mathrm{C}$. The pellets (low-LPS OMVs) obtained were suspended in LPS-free PBS. The low-LPS OMVs were diluted, and the amounts of LPS present in the samples were determined from the developed color intensity using a standard curve constructed with E. coli (011: B4)-derived LPS (concentration 0-1 EU/ml) using limulus amebocyte lysate chromogenic endotoxin quantitation kit, according to manufacturer's protocol. The endotoxin-free water in the kit served as control. One endotoxin unit of LPS per milliliter (EU/ml) equals $0.1 \mathrm{ng}$ endotoxin/ml solution.

Preparation of Macrophage-Derived Conditioned Medium. Primary macrophages were prepared according to a previous report (Liu et al., 2011) with minor modifications. In brief, untreated male SD rats (250-300 g, $n=3)$ were sacrificed by cervical dislocation, and $15 \mathrm{ml}$ PBS was injected i.p. After abdominal massage for 1 minute, PBS-containing peritoneal macrophages were collected and centrifuged at $500 \mathrm{~g}$ for 5 minutes, and pellets were washed with PBS and then cultured in six-well plates $\left(2 \times 10^{6} \%\right.$ well $)$ for 3 hours. After being washed twice with PBS, macrophages were cultured in serum-free DMEM and stimulated with $50 \mu \mathrm{g} / \mathrm{ml}$ OMVs or OMV fractions from normal or Colitis groups for 24 hours. Cells were washed twice with PBS to remove OMVs or fractions and cultured in fresh serum-free DMEM for another 6 hours. The culture medium was centrifuged, and the supernatant was collected as conditioned medium (CM) for experiments. Levels of TNF- $\alpha$, IL- $1 \beta$, IL- 6 , IL- 4 , IL- 10 , IFN- $\gamma$, TGF- $\beta 1$, and MCP-1 in CM were assayed using ELISA kits, according to manufacturer's instruction.

Caco-2 Cell Culture and Treatments. Caco-2 cells at passage 19 were a gift of Dr. Jianqing Ruan at Department of Pharmaceutical Analysis of Soochow
University (Suzhou, China) and cultured in DMEM supplemented with $10 \%$ fetal bovine serum, $1 \%$ nonessential amino acids and streptomycin $(100 \mathrm{U} / \mathrm{ml})$, and penicillin $(100 \mathrm{U} / \mathrm{ml})$ under an atmosphere of $5 \% \mathrm{CO}_{2}$ and $95 \%$ humidified air at $37^{\circ} \mathrm{C}$ in six-well plates. The medium was changed every 3 days until the cells were grown to confluence. Caco- 2 cells were then challenged with CM for 24 hours or incubated in absence or presence of TAK-242 $(1 \mu \mathrm{M})$ alone for 30 minutes, followed by $50 \mu \mathrm{g} / \mathrm{ml}$ OMVs, different fractions, or low-LPS OMVs for another 24 hours. At the end of the experiments, cells were harvested for PCR or Western blot analysis. Each assay was repeated at least three times.

Transfection of TLR4 siRNA into Caco-2 Cells. Caco-2 cells were grown to $40 \%$ confluence and transfected with TLR4 siRNA (5 nM) for 48 hours using Lipofectamine 2000 reagent following the manufacturer's instructions. The positive control siRNA to human glyceraldehyde-3-phosphate dehydrogenase (GAPDH) was measured to monitor siRNA transfection efficiency by real-time RT-PCR. The siRNA negative control (NC) comprised of a 21-bp nontargeting sequence functions to distinguish sequence-specific silencing from nonspecific effects. After transfection, Caco- 2 cells were treated with $50 \mu \mathrm{g} / \mathrm{ml}$ OMVs or low-LPS OMVs from normal and colitis rats for 24 hours, and cells were collected for Western blot or PCR assay in triplicates. TLR4, GAPDH, and NC siRNA sequences were as follows: TLR4-770, forward primer: 5'-GCUCACAAUCUUAUCCAAUTT-3', reverse primer: 5'-AUUGGAUAAGAUUGUGAGCTT-3'; TLR4-931, forward primer: $5^{\prime}$-CCUGAACCCUAUGAACUUUTT-3', reverse primer: 5'-AAAGUUCAUAGGGUUCAGGTT-3'; TLR4-1240, forward primer: 5'-CCUGGUGAGUGUGACUAUUTT-3', reverse primer: 5' -AAUAGUCACACUCACCAGGTT-3'; NC siRNA, forward primer: 5'-UUCUCCGAACGUGUCACGUTT-3' ${ }^{\prime}$, reverse primer: 5'-ACGUGACACGUUCGGAGAATT-3'; and GAPDH siRNA, forward primer: 5'-UGACCUCAACUACAUGGUUTT-3', reverse primer: 5'-AACCAUGUAGUUGAGGUCATT-3'.

Western Blot Analysis. Small intestinal or colonic mucosa or Caco-2 cells were washed twice with ice-cold PBS and then lysed in Western blot lysis buffer $(50 \mathrm{mM}$ Tris- $\mathrm{HCl}$, pH 7.2, containing $1 \%$ sodium deoxycholate, $0.1 \%$ SDS, $0.15 \mathrm{mM} \mathrm{NaCl}$, $1 \%$ Nonidet P-40, $1 \mathrm{mM}$ sodium orthovanadate) at $4^{\circ} \mathrm{C}$ for 30 minutes. The supernatant was then obtained by centrifugation at $12,000 \mathrm{rpm}$ for 20 minutes at $4^{\circ} \mathrm{C}$, and the protein content was determined using BCA kits. The proteins were separated by $10 \%$ SDS-PAGE and transferred to PVDF membranes by semidry electrophoretic transfer. The PVDF membranes were then blocked with $5 \%$ skim milk in TBST buffer (5 mM Tris-HCl, pH 7.6, $136 \mathrm{mM} \mathrm{NaCl}, 0.05 \%$ Tween 20 ) overnight at $4^{\circ} \mathrm{C}$, followed by incubating with primary antibody $\left(1: 1000\right.$ dilution in TBST) at $4{ }^{\circ} \mathrm{C}$ overnight. The PVDF membrane was washed three times with TBST buffer and incubated with the secondary antibody horseradish peroxidase-labeled anti-rabbit or anti-mouse $\operatorname{IgG}$ (1:2000 dilution in TBST) at room temperature for 1 hour. The signals were detected by using enhanced chemiluminescence detection reagent and semiquantified by densitometry with Image-Pro Plus software.

RT Quantitative PCR Assay. Small intestinal and colonic mucosa, Caco-2 cells, or macrophages were homogenized, and mRNA was extracted using TRIzol Plus. mRNA concentration was calculated from QuantiFluor RNA System. To generate cDNA from mRNA template, $500 \mathrm{ng}$ total mRNA was dissolved in $20 \mu \mathrm{l}$ reaction system $\left[1 \mu \mathrm{l}\right.$ Oligo(dt) ${ }_{18}, 4 \mu 15 \times$ reaction buffer, $1 \mu 1$ RNase inhibitor, $2 \mu \mathrm{l}$ $\mathrm{dNTP}(10 \mathrm{mM})$, and $1 \mu \mathrm{lRT}]$. The mixture were degenerated at $42^{\circ} \mathrm{C}$ for 60 minutes and annealed at $70^{\circ} \mathrm{C}$ for 5 minutes. An aliquot of cDNA ( $4 \mu 1$ RT product) was dissolved in $50 \mu \mathrm{l}$ PCR mixture [26 $\mu 11 \times$ SYBR Green master mix, $1 \mu$ l each primer (final concentration $0.2 \mu \mathrm{mol} / \mathrm{l}$ ), $18 \mu \mathrm{l}$ sterile water]. The target gene primer sequences were provided in Supplemental Tables 1 and 2 . The amplification profile consisted of an initial denaturation at $95^{\circ} \mathrm{C}$ for 30 seconds, 60 cycles of $95^{\circ} \mathrm{C}$ for 5 seconds, and then $60^{\circ} \mathrm{C}$ for 34 seconds. The fluorescence data were collected by ViiA7 quantitative PCR instrument at the end of the elongation step per each cycle. The PCR data were analyzed using the $2^{-\Delta \Delta \mathrm{Ct}}$ method to determine the fold changes of relative abundance to internal control gene $\beta$-actin.

Statistical Analysis. All data were expressed as the mean \pm S.D. Significance of the differences between groups was determined by one-way analysis of variance with a Scheffe post hoc test using GraphPad Prism software. Differences were considered statistically significant when $P<0.05$.

\section{Results}

Effect of DSS and Fecal Microbiota Transplantation on Rat Colitis. Seven-day DSS treatment decreased body weight with DAI increasing from day 1 to 7 . DSS induction also caused colonic edema 
and ulcer, shortening, and bleeding, leading to remarkable increase of the colon weight to length ratio in colitic rats. In addition, DSS induction significantly increased the activity of colonic MPO and enhanced the production of both proinflammatory (TNF- $\alpha$, IL- $1 \beta$, and IL-6) and antiinflammatory (IL-4 and IL-10) cytokines from day 3 to 7 (Supplemental Figs. 1 and 2).

FMT from Colitis to normal (NU) rats showed increased DAI from day 4 to 7, colonic edema, elevated MPO activity, and increased pro- and antiinflammatory cytokine levels from day 3 to 7 , although to much less extents than DSS induction. Although the opposite [normal to Colitis (UN)] significantly suppressed the increase of DAI from day 4 to 7 and colonic edema, shortening and bleeding, decreased MPO activity and alleviated the production of both pro- and anti-inflammatory cytokines in NU group (Supplemental Figs. 1 and 2). FMT from normal to normal (NN) rats showed insignificant changes on the above colitis measurements.

Overall, the tissue damage tended to the terminal colon and could be classified as mild to aggravated colitis (Supplemental Fig. 3). DSS induction caused the loss of intestinal crypts with goblet cells, tissue damage on the epithelial layer, and increase of leukocyte infiltration. The NU rats showed similar colonic damage, including increase of leukocyte infiltration and loss of intestinal crypts. FMT from UN rats suppressed the colonic damage induced by DSS. Normal-to-normal FMT showed no colonic damage. The results indicated that DSS-induced gut dysbiosis could elicit colitis-like symptoms that could be abrogated by transplantation of normal fecal microbiota.

mRNA and Protein Expressions of UGT1A1 and NRs along Small Intestine and Colon of Rats. As shown in Fig. 1A, 7-day DSS stimulation significantly increased mRNA expression of rat UGT1A1 (rUGT1A1) in small intestine of Colitis group. Correspondingly, DSS induction resulted in the upregulation of rat PXR (rPXR), rat CAR (rCAR), and rat PPAR (rPPAR)- $\gamma$ in small intestine, whereas significant decreases of rPXR and rCAR in ileum were observed. Unlike the small intestine, colonic mRNA expression of UGT1A1 and NRs were all decreased in colitis rats.

Small intestinal and colonic mRNA expression of UGT1A1 and NRs were unaltered by transplantation of normal feces to normal rats (NN group) (Fig. 1A). Colitis-to-normal FMT (NU group) unaltered small intestinal UGT1A1 expression; however, it caused more severe damage in colon, leading to diminished mRNA expression of rUGT1A1. The opposite (UN group) could partially diminish DSS-induced changes (upregulation in small intestine and downregulation in colon), although at less extents in most cases. Similarly, FMT from colitis rats to normal rats did not affect small intestinal NRs, except for an elevation of rPXR in duodenum, whereas the NRs expression in colon was reduced to an extent less than DSS insult. Oral administration of normal feces to colitis rats (UN group) could abrogate the changes of rPXR and rCAR in jejunum and ileum induced by DSS, but unaffect those in duodenum and colon. Neither did the rPPAR- $\gamma$ expression in both small intestine and colon. The results indicated that gut dysbiosis induced by DSS alters intestinal UGT1A1 and NRs expression at mRNA levels with general elevation in small intestine and reduction in colon.

When the protein expressions of UGT1A1 and NRs were measured, in contrast to the elevation at mRNA levels, DSS induction resulted in the downregulation of rUGT1A1 proteins in duodenum and colon (Fig. 1B). The downregulation of rUGT1A1 and rat NRs (rNRs) was also observed in the whole small intestine (data not shown). The protein expressions of rPXR, rCAR, and rPPAR- $\gamma$ were changed by DSS in the same direction. rUGT1A1 and rNR proteins were unaltered in NN group. In contrast to the unaltered mRNA expression, significant reduction of the protein expressions of rUGT1A1 and rNRs in duodenum and colon was observed in NU group. Transplantation of normal feces to colitis rats (UN group) partly reversed DSS-induced
rUGT1A1 and rNRs downregulation. The results indicated that DSSinduced gut dysbiosis can lead to the downregulation of intestinal UGT1A1 and NRs at protein levels that can be abolished by normal-tocolitis FMT.

mRNA and Protein Expressions of UGT1A1 and NRs in Caco-2 Cells Stimulated by Microbial OMVs and DOC-Treated OMVs. When the microbial OMVs obtained from fecal samples of each group were incubated with Caco- 2 cells, both mRNA and protein expressions of hUGT1A1 were significantly decreased regardless of the origins of OMVs (Fig. 2). OMVs from colitis rats and normal rats receiving FMT from colitis rats reduced the expressions of hUGT1A1 in Caco- 2 cells more significantly than those from their normal counterparts. FMT from normal to colitis rats diminished the effect of colitis OMVs on hUGT1A1 expression in Caco-2 cells. The expression of human PXR (hPXR), human CAR (hCAR), and human PPAR (hPPAR)- $\gamma$ showed similar changes in Caco- 2 cells, all downregulated by OMV stimulation. The inhibitory potency of OMVs on expressions of hUGT1A1 and hNRs is in the same descending order: Colitis $>\mathrm{UN}>\mathrm{NU}>\mathrm{NN} \approx$ normal.

As shown in Supplemental Fig. 4, both normal and colitis OMVs contain LPS, with the LPS level in colitis OMVs significantly higher than that in normal OMVs. DOC treatment successfully reduced LPS from the OMV preparations of both groups to control level (control, $0.662 \mathrm{EU} / \mathrm{ml}$; DOC-treated $\mathrm{N}_{\mathrm{OMV}}, 0.571 \mathrm{EU} / \mathrm{ml}$; DOC-treated coliti$\left.\mathrm{s}_{\mathrm{OMVs}}, 0.497 \mathrm{EU} / \mathrm{ml}\right)$.

When DOC-treated OMV preparations were incubated with Caco-2 cells, protein expressions of hUGT1A1 and hNRs were altered in opposite directions (Fig. 3A): upregulated by that of normal, whereas downregulated by that of colitis, which was less potent than respective complete OMVs. The results indicate that the components other than LPS contribute significantly to the regulatory effects of OMVs on intestinal UGT1A1 and NRs expression.

mRNA Expressions of UGT1A1 and NRs in Caco-2 Cells Treated with CM of OMVs-Stimulated Macrophages. As shown in Fig. 4A, OMVs pretreatment evoked inflammatory responses in macrophages, which resulted in overproduction of both M1-type (TNF- $\alpha$, IL-1 $\beta$, IL-6, MCP-1, IFN- $\gamma$ ) and M2-type (IL-4, IL-10, TGF- $\beta 1$ ) cytokines and chemokines in CM, indicating activation of macrophages by bacterial OMVs. The effects of colitis OMVs were more potent than the normal OMVs.

Macrophages could steer intestinal immune responses through releasing cytokines and chemokines. To determine whether bacterial OMVs-stimulated macrophages contribute to regulation of UGTs/NRs in IECs, Caco- 2 cells were treated with CM of macrophages stimulated with OMVs. Similar to the direct effects of OMVs, the mRNA expression of hUGT1A1 in Caco-2 cells was significantly suppressed accompanying downregulation of the hNRs (Fig. 4B). The CM obtained from colitis OMV-treated macrophages was more potent than that treated by the normal OMVs. The results indicated that OMVs could downregulate UGT1A1 and NRs through evoking inflammation in IECs via promoting macrophage polarization.

Effect of Different OMV Fractions on mRNA Expression of UGT1A1 and NRs in Caco-2 Cells. Bacterial OMVs were further fractionated into five fractions with different molecular weight ranges [ $<3$ (F < 3), 3-10 (F3-10), 10-30 (F10-30), 30-50 (F30-50), >50 kDa $(\mathrm{F}>50)]$, which showed big differences in the protein contents between normal and colitis groups (higher in $<10-\mathrm{kDa}$ fractions and lower in F10-30 and F $>50$ fractions of colitis group than the normal group; Supplemental Fig. 5), indicating that DSS induction caused marked changes of bacterial compositions and/or cellular components. The $\mathrm{F}<3$ fractions of both normal and colitis groups showed no effect on hUGT1A1/hNR mRNA expressions (Fig. 5A). F10-30 fractions 
A

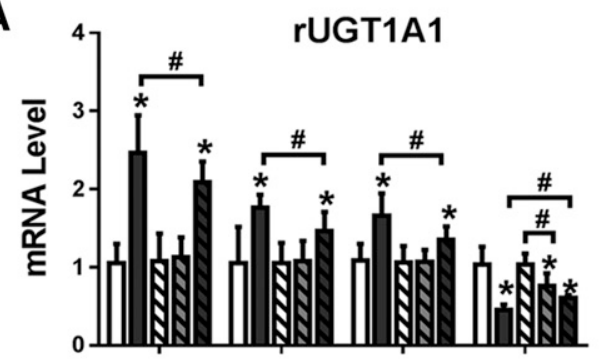

rCAR

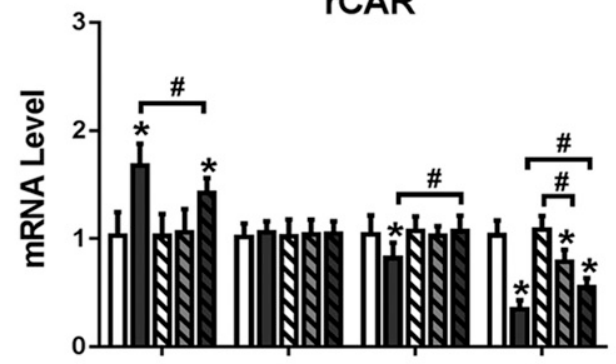<smiles>C[13CH][13CH]</smiles>

B
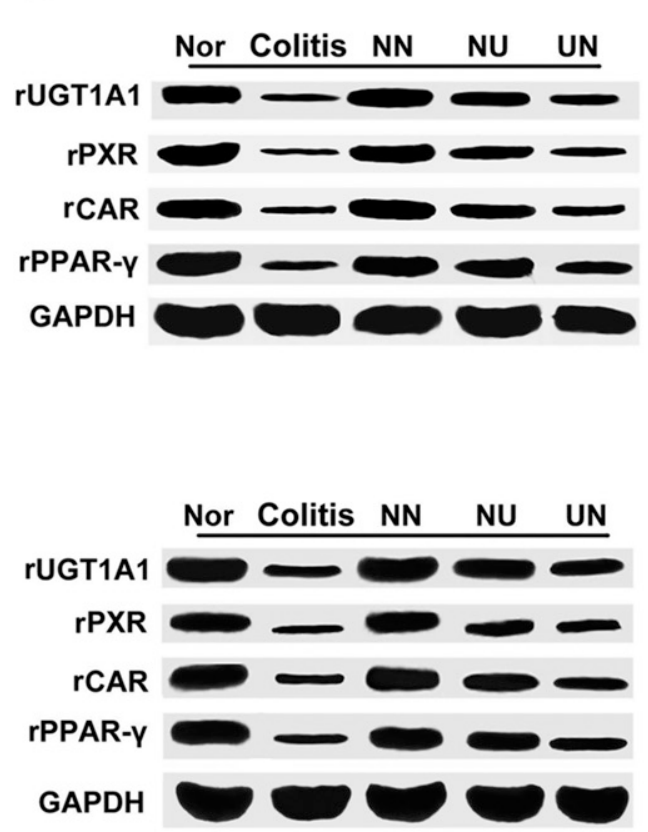

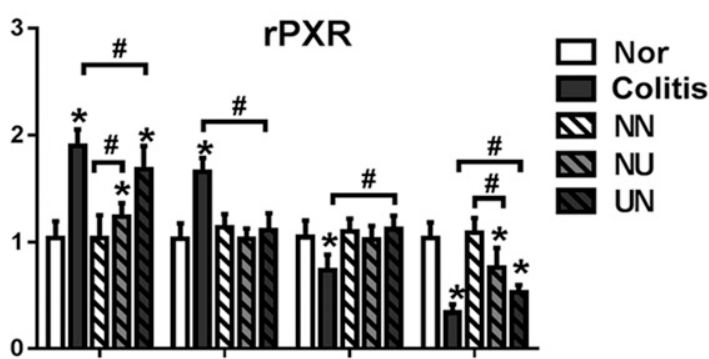

rPPAR-y

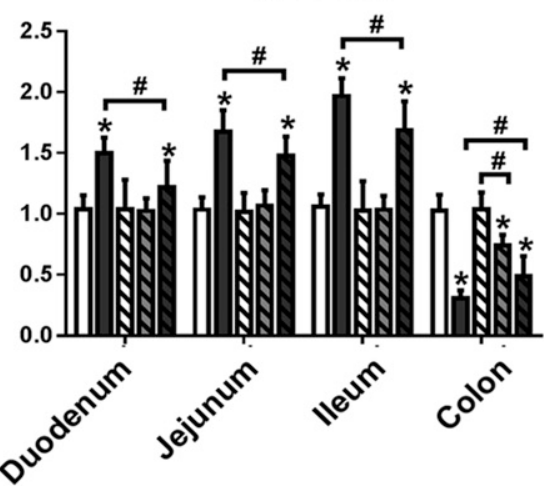

Duodenum

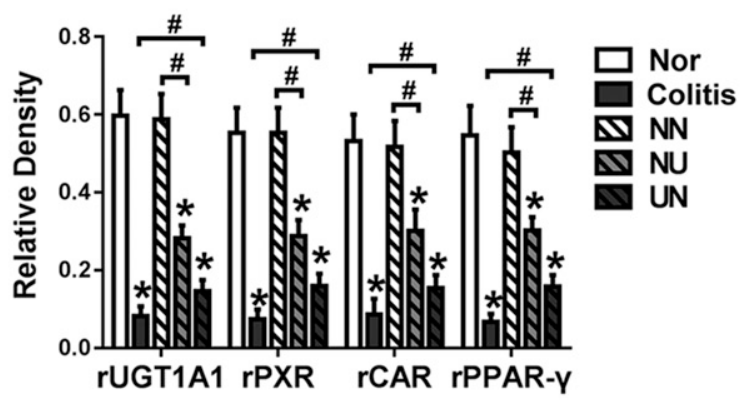

Colon

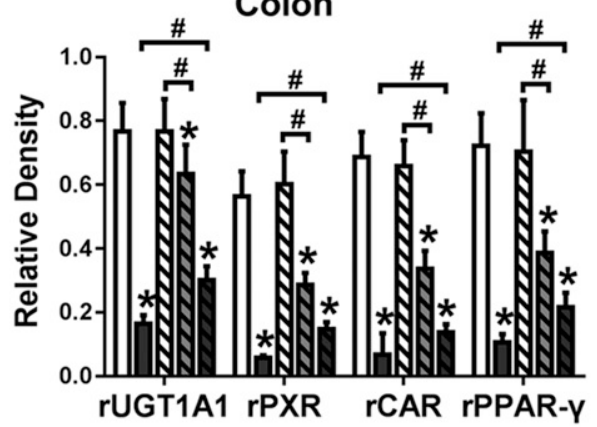

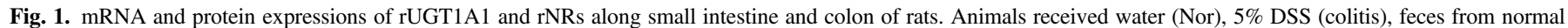

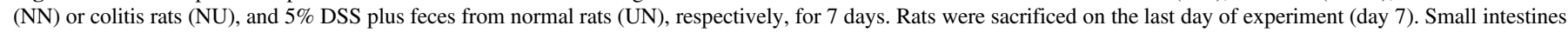

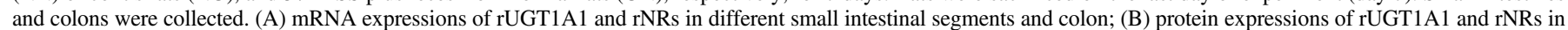

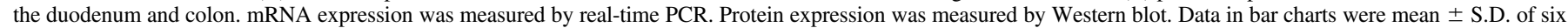

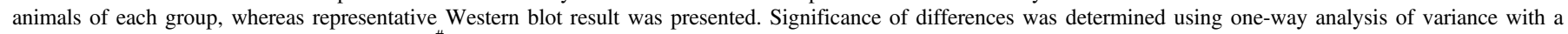
Scheffe post hoc test. ${ }^{*} P<0.05$ versus Nor; ${ }^{\#} P<0.05$ between specific two groups compared.

significantly suppressed hUGT1A1 expression, colitis group showing more potent effects. The F30-50 fractions of both groups reduced hUGT1A1 expression to similar extents. Interestingly, a significant increase of hUGT1A1 was observed with the $\mathrm{F}>50$ fraction of normal group, whereas the fraction of colitis group decreased it significantly. Similarly, the F3-10 fraction of normal group did not affect the mRNA expression of all three hNRs, whereas that of the colitis group significantly suppressed hPXR and hCAR expression (Fig. 5A). The F10-30 and F30-50 fractions of both groups significantly downregulated hPXR and hPPAR- $\gamma$, with those of colitis group showing more potent effects. It is interesting to note that the $\mathrm{F}>50$ fraction of normal OMVs unaltered hPXR and enhanced hCAR, while 

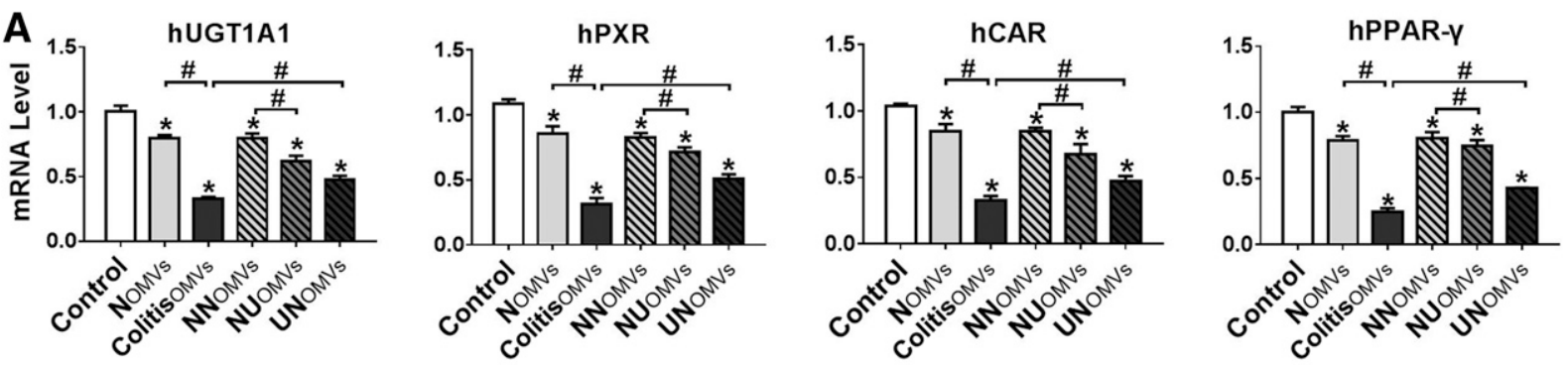

B
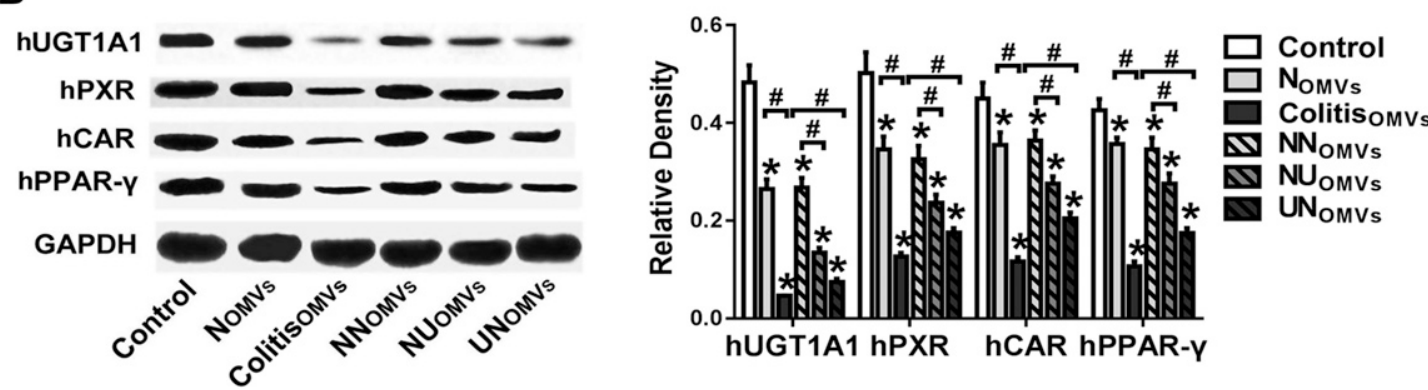

Fig. 2. mRNA and protein expressions of hUGT1A1 and hNRs in Caco-2 cells stimulated by microbial OMVs. Cells were treated with or without $50 \mu \mathrm{g} / \mathrm{ml}$ OMVs from normal, colitis, NN, NU, or UN rat feces for 24 hours. (A) mRNA expression was measured by real-time PCR; (B) protein expression was measured by Western blot. Data in bar charts were mean \pm S.D. of triplicate determinations, whereas representative Western blot result was presented. Significance of differences was determined using oneway analysis of variance with a Scheffe post hoc test. ${ }^{*} P<0.05$ versus control; ${ }^{\#} P<0.05$ between specific two groups compared.

suppressing hPPAR- $\gamma$ expression. In contrast, that of colitis OMVs downregulated all three NRs.

Those CM treated by $>10-\mathrm{kDa}$ fractions (F10-30, F30-50, and $\mathrm{F}>50)$ of both normal and colitis OMVs inhibited hUGT1A1 expressions, with more potent effects observed with those treated by colitis OMVs (Fig. 5B). The effects of CM on hNR expression showed similar tendency, and the suppressing effect generally increased with mol. wt. of the OMVs fractions. The highest inhibition was observed with the CM treated by the F30-50 of the colitis OMVs, which inhibited the mRNA expression of hPXR to one third of the control cells and half of the cells treated by its normal counterpart.

Expressions of TLR2, TLR4, and NF-אB in Small Intestine and Colon from Rats. As shown in Fig. 6, DSS induction resulted in the upregulation of protein levels of TLR2, TLR4, and NF- $\kappa$ B in small intestine and colon. Normal-to-normal FMT (NN group) unaffected the levels of the three proteins. Normal recipients of colitis fecal samples
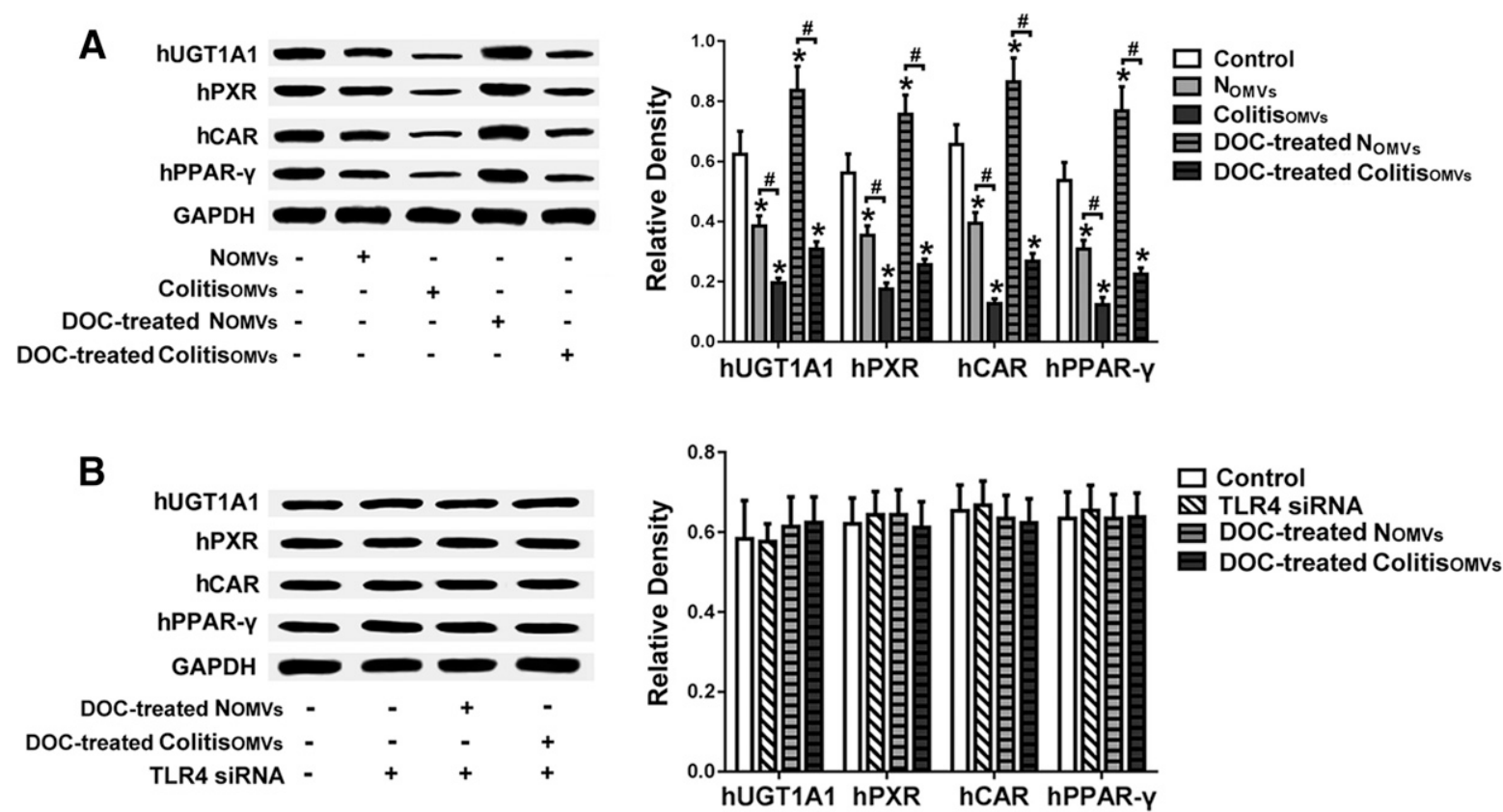

Fig. 3. Protein expressions of hUGT1A1 and hNRs in Caco-2 cells treated by DOC-treated OMVs. (A) Cells were treated with $50 \mu \mathrm{g} / \mathrm{ml}$ DOC-treated OMVs or (B) transfected with TLR4 siRNA, followed by treatment of $50 \mu \mathrm{g} / \mathrm{ml}$ DOC-treated OMVs from normal and colitis feces for 24 hours. Protein expressions were determined by Western blot. Data in bar charts were mean \pm S.D. of triplicate determinations, whereas representative Western blot result was presented. Significance of differences was determined using one-way analysis of variance with a Scheffe post hoc test. ${ }^{*} P<0.05$ versus control; ${ }^{\#} P<0.05$ between specific two groups compared. 
A M1-type
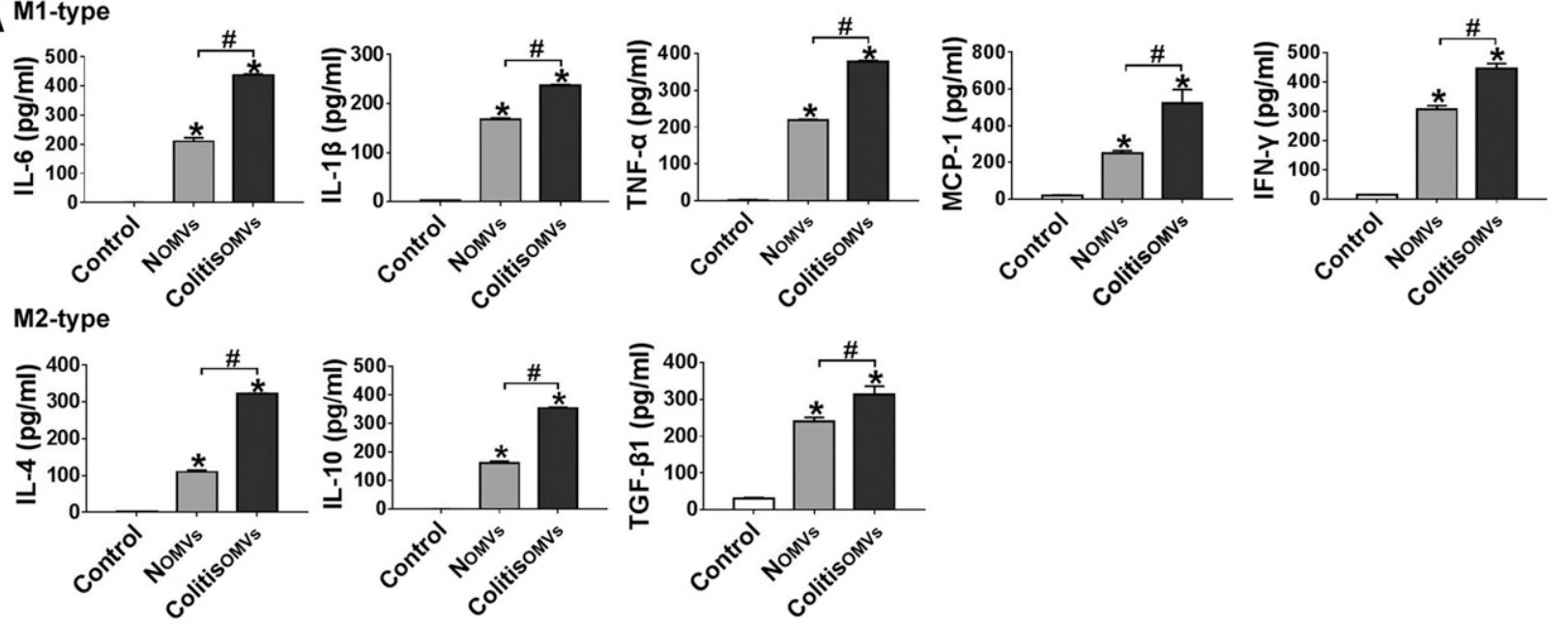

B
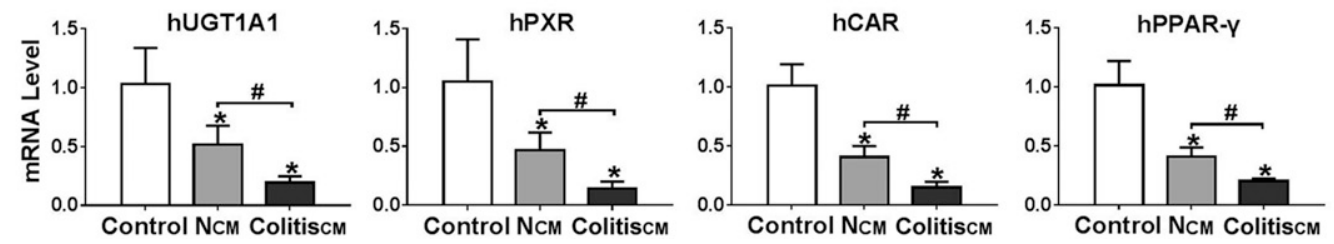

Fig. 4. mRNA expressions of hUGT1A1 and hNRs in Caco-2 cells treated with CM of macrophages. Primary macrophages were treated with $50 \mu \mathrm{g} / \mathrm{ml}$ OMVs from normal and colitis groups for 24 hours. After incubation, macrophages were washed twice with PBS to remove OMVs and cultured in fresh serum-free DMEM for another 6 hours. The culture medium served as CM and collected for ELISA. Caco-2 cells were stimulated with CM. (A) M1-type and M2-type cytokines and chemokines produced in CM; (B) mRNA expressions of hUGT1A1 and hNRs in Caco-2 cells treated by CM. mRNA were measured by real-time PCR. Data were expressed as mean \pm S.D. of triplicate determinations. Significance of differences was determined using one-way analysis of variance with a Scheffe post hoc test. ${ }^{*} P<0.05$ versus control; ${ }^{\#} P<0.05$ between specific groups compared.

(NU group) showed significantly higher protein expression of these proteins than NN group. The FMT from normal to colitis rats partially suppressed the elevation induced by DSS, which, however, was still significantly higher than normal, NN and NU groups. The results indicated that DSS-induced gut dysbiosis can alter the protein expressions of TLR2, TLR4, and NF- $\kappa \mathrm{B}$ in small intestine and colon, and transplantation of normal fecal microbiota can diminish the changes.
Effects of TLR4 Inhibition on mRNA Expression of UGT1A1 and NRs in Caco-2 Cells Treated by OMVs and DOC-Treated OMVs. As shown in Supplemental Fig. 6, when treated by OMVs from normal or colitis rats, TLR2 protein expression in Caco- 2 cells was unaltered, whereas TLR4 protein level was elevated, with colitis OMVs showing more potent effect. The results indicated that TLR4 might be the main signaling molecule mediating OMVs stimulation.
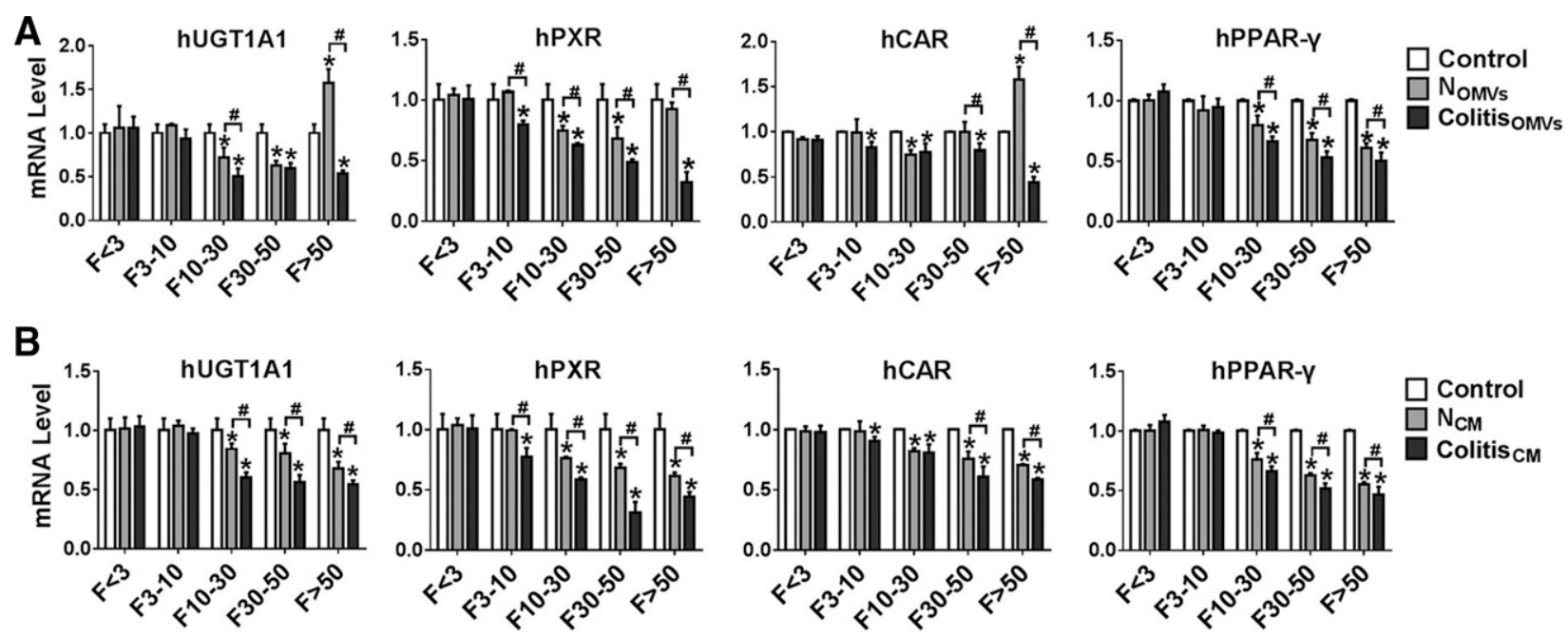

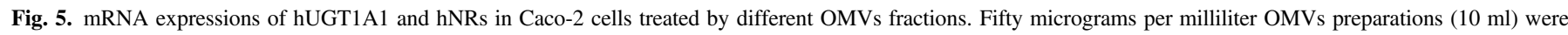

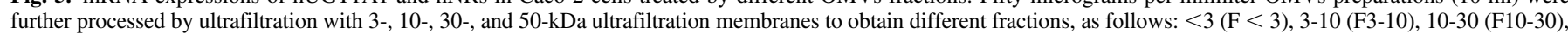

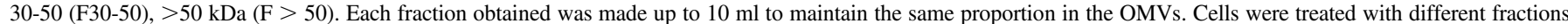

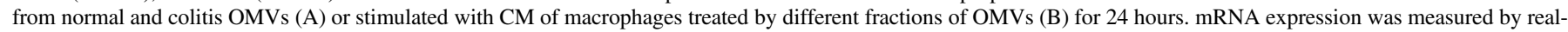

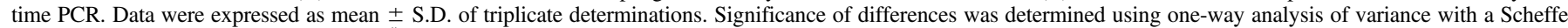
post hoc test. ${ }^{*} P<0.05$ versus control; ${ }^{\#} P<0.05$ between specific two groups compared. 


\section{Small intestine}
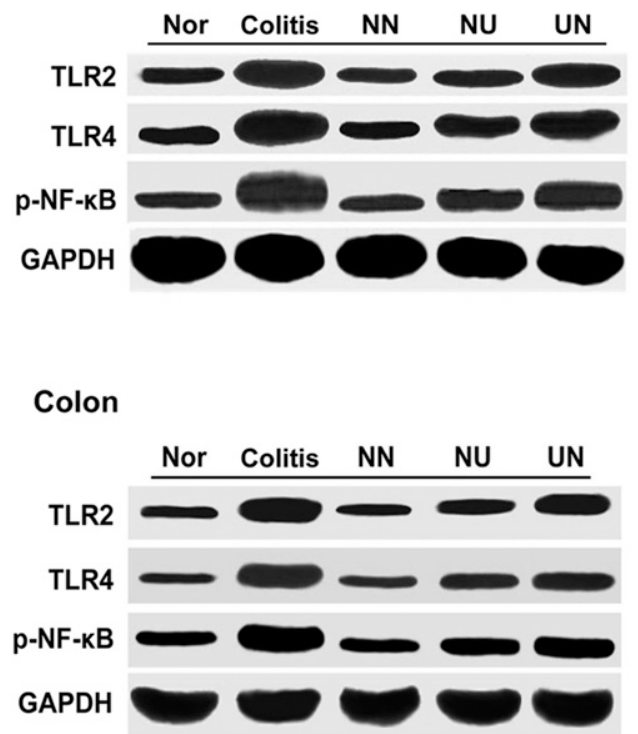
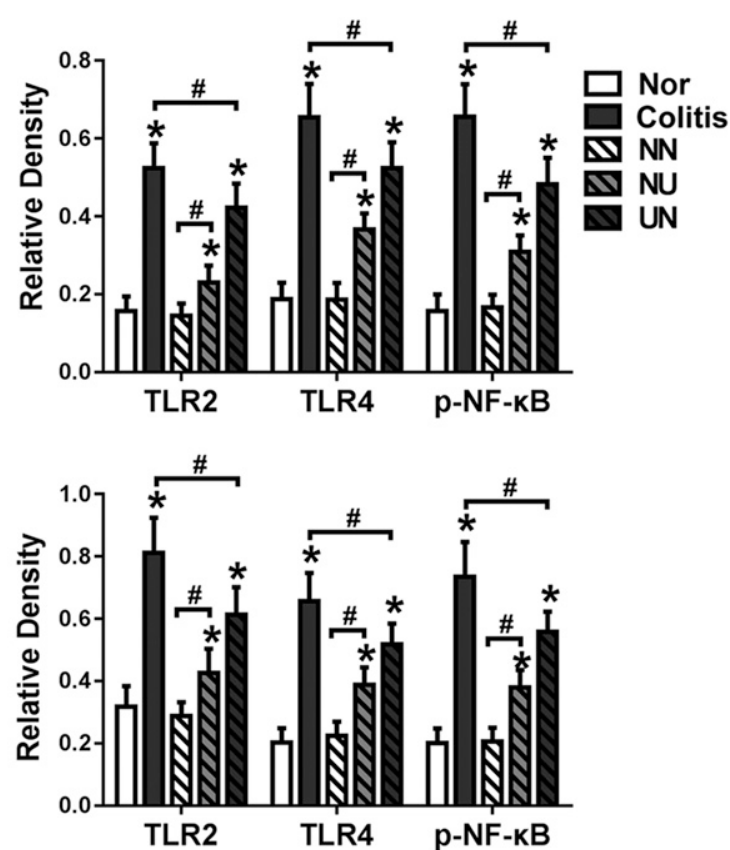

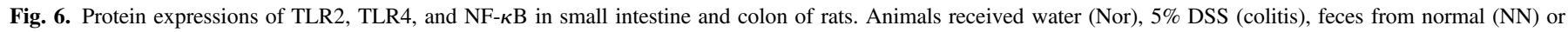

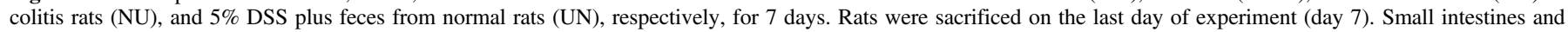

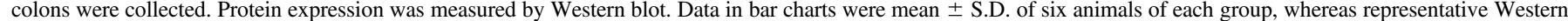

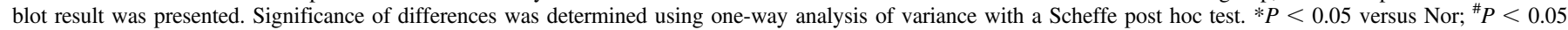
between specific two groups compared.

The presence of TAK-242, a specific inhibitor of TLR4, could partially reverse the downregulation of hUGT1A1 and hNRs by microbial OMVs from both normal and colitis groups at the tested concentration $(1 \mu \mathrm{M})$ of the inhibitor (Supplemental Fig. 7). When Caco- 2 cells were transfected with TLR4 siRNA, the downregulation of hUGT1A1 and hNRs expression by OMVs from both groups was completely abrogated (Fig. 7A). Knocking down TLR4 also abrogated the dysregulation of hUGT1A1 and hNRs expressions by DOC-treated OMVs from both normal and colitis feces (Fig. 3B). These results indicated that both complete OMVs and low-LPS OMVs regulate intestinal UGT1A1 and NRs mainly through TLR4 activation.

Effects of TLR4 Inhibition on Phosphorylation of PI3K/Akt and ERK1/2 in Caco-2 Cells Treated by OMVs. Knocking down TLR4 in Caco- 2 cells resulted in a decrease of TLR 4 protein expression as well as reduction of phosphorylation of PI3K/Akt, ERK1/2, and NF- $\kappa \mathrm{B}$ (Fig. 7B). When Caco-2 cells were stimulated by microbial OMVs, TLR4 protein expression was significantly elevated, and so did the phosphorylated PI3K/Akt, ERK1/2, and NF- $\kappa$ B. The colitis OMVs showed more significant effects than the normal OMVs. The TLR4 activation by microbial OMVs from both groups was successfully blocked by TLR4 siRNA transfection, leading to decreased TLR4 expression and diminished phosphorylation of PI3K/Akt, ERK1/2, and NF- $\kappa$ B. These results indicated that OMVs regulated intestinal UGT1A1 and NRs through activating TLR4 and PI3K/Akt and ERK1/2 phosphorylation, which resulted in NF- $\kappa$ B activation.

\section{Discussion}

UGT1A1 serves as an important constitute of intestinal epithelial barrier. Growing evidence links gut dysbiosis with UGT1A1 dysfunction. In this study, we verified the regulatory role of gut microbiota on intestinal UGT1A1 using DSS-induced colitis rat model plus FMT and pinpointed the main molecular events in vitro using bacterial OMVs. The main findings include the following: 1) DSS induced dysregulation of UGT1A1/NRs in rat intestine; 2) colitis-to-normal FMT caused similar alterations of UGT1A1/NRs, whereas the opposite alleviated DSS-induced changes; 3 ) complete OMVs from both normal and colitis rats downregulated UGT1A1/NRs expressions in Caco-2 cells directly as well as via a macrophage-mediated mechanism; low-LPS OMVs from normal rats elicited direct opposite effects to that from colitis rats; and 4) knocking down TLR4 blocked UGT1A1/NRs dysregulation evoked by OMVs and low-LPS OMVs.

DSS-induced rat colitis highly resembles human UC and is widely used in basic research and drug discovery. The microbial shifts in the experimental colitis are similar to those in UC patients (Giaffer et al., 1991; Munyaka et al., 2016). In this study, colitis-to-normal FMT caused similar clinical changes and microbial shifts (data not shown) to DSS induction, whereas FMT in opposite direction partially reversed DSSinduced changes, demonstrating the involvement of gut microbiota in colitis development and therapy.

We found that both UGT1A1 and its upstream transcriptional regulator NRs were changed by DSS-induced colitis, which can be alleviated by normal-to-colitis FMT, demonstrating a regulatory role of gut microbiota in intestinal UGT1A1/NRs. It is interesting to note that normal-to-normal FMT unaffected UGT1A1 and NRs in all cases, and colitis-to-normal FMT (NU group) only caused increase of PXR. These findings indicate that healthy gut microbiota are highly resistant to colonization of those microbial foreigners to maintain intestinal homeostasis (Lawley and Walker, 2013) and also have greater colonization capability than those from colitis rats.

Surprisingly, the mRNA of intestinal rUGT1A1 and rNRs was generally increased in small intestine and decreased in colon by DSS and normal-to-colitis FMT, whereas their proteins were all decreased in duodenum, whole small intestine, and colon. The discrepancy between mRNA and protein levels of rUGT1A1/rNRs in vivo might be due to the following: 1) the existence of other compensatory mechanism in intestinal UGT1A1/NRs regulation, 2) more complicated post-transcriptional mechanisms involved in translating mRNA 

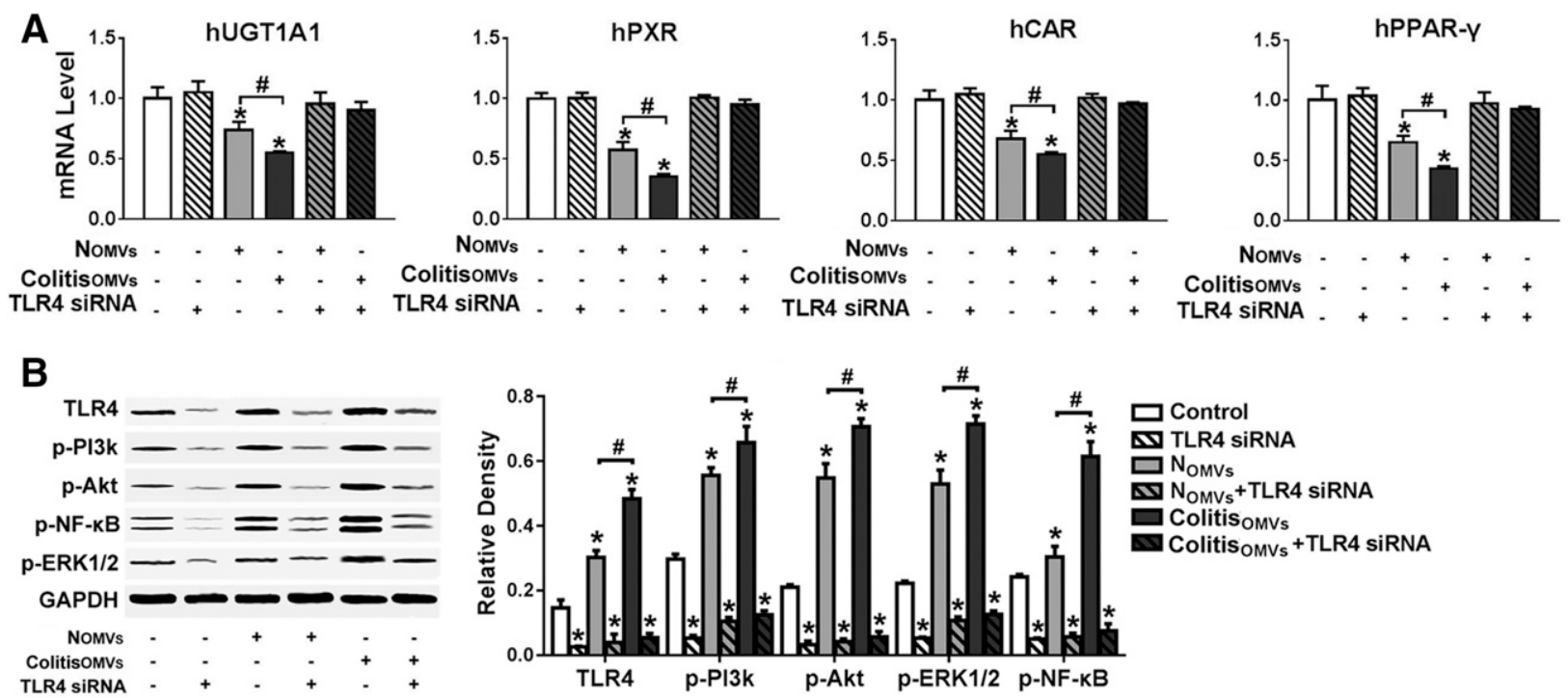

Fig. 7. mRNA expressions of hUGT1A1 and hNRs and protein expressions of phosphorylated PI3K/Akt, ERK1/2, and NF- $\kappa$ B in Caco- 2 cells treated by TLR4 siRNA. Cells were transfected with TLR4 siRNA, followed by treatment of $50 \mu \mathrm{g} / \mathrm{ml}$ OMVs for 24 hours. (A) mRNA expressions of hUGT1A1 and hNRs were determined by realtime PCR; (B) protein expressions of phosphorylated PI3K/Akt, ERK1/2, and NF- $\kappa$ B were determined by Western blot. Data in bar charts were mean \pm S.D. of triplicate determinations, whereas representative Western blot result was presented. Significance of differences was determined using one-way analysis of variance with a Scheffe post hoc test. $* P<0.05$ versus control; ${ }^{\#} P<0.05$ between specific two groups compared.

into proteins (Greenbaum et al., 2003), and 3) the mRNA elevation is the consequence and the earlier event of the regulatory feedback of downregulated proteins. Additionally, colon is the main colonization site of gut microbiota, explaining the more serious colonic mucosal injury. This may also account for the mRNA level discrepancy between small intestine and colon.

Nuclear xenobiotic receptors are essential regulators of drugmetabolizing enzymes and transporters (Ou et al., 2010). This study showed that intestinal rUGT1A1 mRNA was differentially regulated by gut microbiota through dysregulating the rNRs. The changes of rUGT1A1 mRNA were consistent with that of rPPAR- $\gamma$ along small intestine, rPXR and rCAR in duodenum, and all three rNRs in colon, suggesting a tissue-specific regulation of UGT1A1 by NRs. UGT1A1 was relatively unaffected in small intestine, although downregulated in colon in 2,4,6-trinitrobenzenesulfonic acid-treated rats (Zhou et al., 2013). The discrepancy could be due to different mechanisms involved in 2,4,6-trinitrobenzenesulfonic acid- and DSS-induced colitis, which were believed to resemble human Crohn's disease and UC, respectively (Alex et al., 2009). They also observed decreased mRNA levels of PXR and PPAR- $\gamma$ in both small intestine and colon, whereas CAR was unaffected (Zhou et al., 2013). Our study first reported the correlation between intestinal PPAR- $\gamma$ and UGT1A1 at both mRNA and protein levels, indicating the involvement of PPAR- $\gamma$ in regulating intestinal UGT1A1.

Gram-negative bacteria dominated microbial alterations of human UC (Vigsnæs et al., 2012; Kaparakis-Liaskos and Ferrero, 2015). We first addressed the role of Gram-negative bacterial OMVs in intestinal UGT1A1 regulation. hUGT1A1 and hNRs in Caco-2 cells were downregulated at both mRNA and protein levels by all bacterial OMVs preparations, following same descending order of Colitis $>\mathrm{UN}>$ $\mathrm{NU}>\mathrm{NN} \approx$ normal, regardless of their origins. These results agree with the protein level changes in vivo. The mRNA level in vivo-in vitro discrepancy could be attributed to the following: 1) other host factors involved in UGT1A1/NR mRNA regulation, 2) stronger microbial invasion and pathogenic abilities in vitro than in vivo (Jandik et al., 2008), and 3) regulatory feedback on mRNA expression did not occur in vitro due to shorter incubation ( 24 hours versus 7 days) and/or simpler biologic system (cells versus whole organism). OMVs from colitis or NU group downregulated UGT1A1/NRs in Caco-2 cells more potently. This should be attributed to different microbial compositional changes induced by DSS and FMT.

The intestine harbors largest population of macrophages that steer immune responses through releasing cytokines and chemokines (Nakata et al., 2013). In this study, both M1- and M2-type cytokines and chemokines were overproduced by OMV-stimulated Caco- 2 cells, and colitis OMVs showed stronger effects, supporting that gut dysbiosis causes macrophage polarization and enhances proinflammatory responses in IECs. Incubation of Caco-2 cells with CM from OMVtreated rat primary macrophages resulted in similar changes of UGT1A1/NRs, which were inversely proportional to cytokine/ chemokine production. Taken together, the in vitro data support that microbial OMVs regulate intestinal UGT1A1/NRs directly and via a macrophage-mediated mechanism.

We further tried to locate the major effector molecules of OMVs. In general, those $>10-\mathrm{kDa}$ fractions from both normal and colitis OMVs significantly decreased hUGT1A1 and hNRs, and, in most cases, colitis OMVs were more potent than the normal counterparts. Notably, the $>50-\mathrm{kDa}$ fractions from normal OMVs upregulated hUGT1A1 and hCAR, comparing to a downregulation by colitis OMVs. CM from macrophages treated by different OMV fractions caused similar changes. The differential regulatory effects of normal and colitis OMVs and fractions on hUGT1A1 and hNRs should be a result of microbial compositional shifts and/or metabolic capability alterations induced by DSS. Even though we observed some correlations between the protein contents and the effects, for example, higher protein content of the $>50$ $\mathrm{kDa}$ fractions of normal group correlates with their upregulatory effects on hUGT1A1 and hCAR, we could not rule out the involvement of other components in the fraction, and the constitute proteins may also vary with sample. However, the chemical complexity of microbial OMVs and the analytical bottleneck for complex biologic samples hamper the identification of the molecular effectors.

TLR4 was significantly increased in IECs of UC patients (Cario and Podolsky, 2000). TLR4 pathway disturbance is implicated in UC development. TLR2 deficiency triggers early tight junction disruption, 
which aggravates colonic inflammation (Cario et al., 2007). In this study, both TLR2 and TLR4 were upregulated in small intestine and colon of colitis, NU, and UN rats, and activated by total bacterial preparations from normal and colitis groups (Supplemental Fig. 8). However, only TLR4 was significantly activated by OMVs in vitro. These findings are in line with a previous report that TLR2 and TLR4 mainly sense Gram-positive and Gram-negative bacterial signals, respectively (Takeuchi et al., 1999). Knocking down TLR4 abrogated hUGT1A1/hNRs downregulation by OMVs, confirming TLR4 as the main mediator of bacterial OMVs signaling. However, further study is needed to determine the role of TLR2 in intestinal UGT1A1/NRs regulation in vivo and whether it accounts for the mRNA level in vivo-in vitro differences.

PI3K/Akt and mitogen-activated protein kinase (MAPK) are two major downstream pathways of TLR4 and play critical roles in various cellular processes (Troutman et al., 2012; Peroval et al., 2013). NF- $\kappa$ B is a key mediator of inflammatory responses. TLR4 signaling leads to rapid activation of PI3K and phosphorylation of PI3K downstream targets Akt and ERK1/2, leading to NF- $\kappa \mathrm{B}$ activation and proinflammatory cytokine production. MAPK signaling pathway activation also impacts NF- $\kappa$ B activation (Remels et al., 2009). NF- $\kappa$ B activation was shown to inhibit PXR, CAR, and PPAR- $\gamma$, and, vice versa, NF- $\kappa$ B inhibition could enhance these NR activities (Shah et al., 2007; Necela et al., 2008; Chai et al., 2013). We also observed a negative correlation between $\mathrm{NF}-\kappa \mathrm{B}$ and the NRs. OMVs stimulated TLR4, resulting in NF- $\kappa \mathrm{B}$ activation as well as PI3K/Akt and ERK1/2 phosphorylation in Caco-2 cells, which was abolished by TLR4 siRNA transfection, supporting that OMVs downregulate intestinal UGT1A1 through decreasing NRs by TLR4 activation via MAPK/ERK and PI3K/Akt pathways.

LPS was considered to be the major contributor to virulence and inflammatory responses of Gram-negative bacteria. To determine whether other components in OMVs contribute to intestinal UGT1A1/ NRs regulation, we used DOC to prepare low-LPS OMVs (decreased from hundreds to $<1 \mathrm{EU} / \mathrm{ml}$ ). In contrast to the decreases of hUGT1A1/ hNRs by complete OMVs, the low-LPS OMV preparations of normal rats upregulated hUGT1A1/NRs through TLR4. This might account for the upregulation of hUGT1A1/hCAR by the normal $\mathrm{F}>50$ fraction. The low-LPS colitis OMVs decreased hUGT1A1/hNR expression, although less potent than respective complete OMVs. These data support the involvement of other OMVs components in intestinal UGT1A1/NRs regulation, demonstrating that OMVs are more suitable than LPS as the study materials for investigating host-bacteria interactions.

In conclusion, this study has demonstrated a regulatory role of gut microbiota on intestinal UGT1A1 and NRs. Gram-negative bacterial OMVs exhibited general downregulation through directly interacting with host IECs via TLR4 activation and inducing macrophage polarization, offering new insights into intestinal UGT1A1 dysfunction in gut dysbiosis-related diseases.

\section{Authorship Contributions}

Participated in research design: Gao, R. Yan.

Conducted experiments: Gao, Li, Wei, Z.-X. Yan, Hu, Huang, Han, Wai, Yang.

Performed data analysis: Gao, Li, R. Yan.

Wrote or contributed to the writing of the manuscript: Gao, R. Yan.

\section{References}

Alex P, Zachos NC, Nguyen T, Gonzales L, Chen TE, Conklin LS, Centola M, and Li X (2009) Distinct cytokine patterns identified from multiplex profiles of murine DSS and TNBS-induced colitis. Inflamm Bowel Dis 15:341-352.

Beutler B and Rietschel ET (2003) Innate immune sensing and its roots: the story of endotoxin. Nat Rev Immunol 3:169-176.
Borody TJ, Paramsothy S, and Agrawal G (2013) Fecal microbiota transplantation: indications, methods, evidence, and future directions. Curr Gastroenterol Rep 15:337.

Cario E, Gerken G, and Podolsky DK (2007) Toll-like receptor 2 controls mucosal inflammation by regulating epithelial barrier function. Gastroenterology 132:1359-1374

Cario E and Podolsky DK (2000) Differential alteration in intestinal epithelial cell expression of Toll-like receptor 3 (TLR3) and TLR4 in inflammatory bowel disease. Infect Immun 68: 7010-7017.

Chai X, Zeng S, and Xie W (2013) Nuclear receptors PXR and CAR: implications for drug metabolism regulation, pharmacogenomics and beyond. Expert Opin Drug Metab Toxicol 9: 253-266.

Christensen H and Hermann M (2012) Immunological response as a source to variability in drug metabolism and transport. Front Pharmacol 3:8.

Claassen I, Meylis J, van der Ley P, Peeters C, Brons H, Robert J, Borsboom D, van der Ark A, van Straaten I, Roholl P, et al. (1996) Production, characterization and control of a Neisseria meningitidis hexavalent class 1 outer membrane protein containing vesicle vaccine. Vaccine 14: 1001-1008.

Cosín-Roger J, Ortiz-Masiá D, Calatayud S, Hernández C, Alvarez A, Hinojosa J, Esplugues JV, and Barrachina MD (2013) M2 macrophages activate WNT signaling pathway in epithelial cells: relevance in ulcerative colitis. PLoS One 8:e78128.

Eddy JL, Gielda LM, Caulfield AJ, Rangel SM, and Lathem WW (2014) Production of outer membrane vesicles by the plague pathogen Yersinia pestis. PLoS One 9:e107002.

Ellis TN and Kuehn MJ (2010) Virulence and immunomodulatory roles of bacterial outer membrane vesicles. Microbiol Mol Biol Rev 74:81-94.

Ellis TN, Leiman SA, and Kuehn MJ (2010) Naturally produced outer membrane vesicles from Pseudomonas aeruginosa elicit a potent innate immune response via combined sensing of both lipopolysaccharide and protein components. Infect Immun 78:3822-3831.

Ghia JE, Galeazzi F, Ford DC, Hogaboam CM, Vallance BA, and Collins S (2008) Role of M-CSF-dependent macrophages in colitis is driven by the nature of the inflammatory stimulus. Am J Physiol Gastrointest Liver Physiol 294:G770-G777.

Giaffer MH, Holdsworth CD, and Duerden BI (1991) The assessment of faecal flora in patients with inflammatory bowel disease by a simplified bacteriological technique. J Med Microbiol 35: 238-243.

Greenbaum D, Colangelo C, Williams K, and Gerstein M (2003) Comparing protein abundance and mRNA expression levels on a genomic scale. Genome Biol 4:117.

Huang Y, Hu N, Gao X, Yan Z, Li S, Jing W, and Yan R (2015) Alterations of testosterone metabolism in microsomes from rats with experimental colitis induced by dextran sulfate sodium. Chem Biol Interact 232:38-48.

Jandik KA, Bélanger M, Low SL, Dorn BR, Yang MC, and Progulske-Fox A (2008) Invasive differences among Porphyromonas gingivalis strains from healthy and diseased periodontal sites. J Periodontal Res 43:524-530.

Kaparakis-Liaskos M and Ferrero RL (2015) Immune modulation by bacterial outer membrane vesicles. Nat Rev Immunol 15:375-387.

Kim JH, Yoon YJ, Lee J, Choi EJ, Yi N, Park KS, Park J, Lötvall J, Kim YK, and Gho YS (2013) Outer membrane vesicles derived from Escherichia coli up-regulate expression of endothelial cell adhesion molecules in vitro and in vivo. PLoS One 8:e59276.

Kono Y, Miyoshi S, and Fujita T (2016) Dextran sodium sulfate alters cytokine production in macrophages in vitro. Pharmazie 71:619-624.

Lawley TD and Walker AW (2013) Intestinal colonization resistance. Immunology 138:1-11

Li J, Butcher J, Mack D, and Stintzi A (2015) Functional impacts of the intestinal microbiome in the pathogenesis of inflammatory bowel disease. Inflamm Bowel Dis 21:139-153.

Liu K, Luo T, Zhang Z, Wang T, Kou J, Liu B, and Huang F (2011) Modified Si-Miao-San extract inhibits inflammatory response and modulates insulin sensitivity in hepatocytes through an IKK $\beta /$ IRS-1/Akt-dependent pathway. J Ethnopharmacol 136:473-479.

Lu YC, Yeh WC, and Ohashi PS (2008) LPS/TLR4 signal transduction pathway. Cytokine 42:145-151. Marks DJ and Segal AW (2008) Innate immunity in inflammatory bowel disease: a disease hypothesis. J Pathol 214:260-266.

Mirlashari MR, Høiby EA, Holst J, and Lyberg T (2001) Outer membrane vesicles from Neisseria meningitidis: effects on cytokine production in human whole blood. Cytokine 13:91-97.

Morgan ET, Goralski KB, Piquette-Miller M, Renton KW, Robertson GR, Chaluvadi MR, Charles KA, Clarke SJ, Kacevska M, Liddle C, et al. (2008) Regulation of drug-metabolizing enzymes and transporters in infection, inflammation, and cancer. Drug Metab Dispos 36:205-216.

Munyaka PM, Rabbi MF, Khafipour E, and Ghia JE (2016) Acute dextran sulfate sodium (DSS)induced colitis promotes gut microbial dysbiosis in mice. J Basic Microbiol 56:986-998.

Nakata K, Yamamoto M, Inagawa H, and Soma G (2013) Effects of interactions between intestinal microbiota and intestinal macrophages on health. Anticancer Res 33:2849-2853.

Necela BM, Su W, and Thompson EA (2008) Toll-like receptor 4 mediates cross-talk between peroxisome proliferator-activated receptor gamma and nuclear factor-kappaB in macrophages. Immunology 125:344-358.

Ou Z, Huang M, Zhao L, and Xie W (2010) Use of transgenic mice in UDPglucuronosyltransferase (UGT) studies. Drug Metab Rev 42:123-131.

Park KS, Lee J, Jang SC, Kim SR, Jang MH, Lötvall J, Kim YK, and Gho YS (2013) Pulmonary inflammation induced by bacteria-free outer membrane vesicles from Pseudomonas aeruginosa. Am J Respir Cell Mol Biol 49:637-645.

Peroval MY, Boyd AC, Young JR, and Smith AL (2013) A critical role for MAPK signalling pathways in the transcriptional regulation of Toll like receptors. PLoS One 8:e51243.

Remels AH, Langen RC, Gosker HR, Russell AP, Spaapen F, Voncken JW, Schrauwen P, and Schols AM (2009) PPARgamma inhibits NF-kappaB-dependent transcriptional activation in skeletal muscle. Am J Physiol Endocrinol Metab 297:E174-E183.

Richardson TA, Sherman M, Kalman D, and Morgan ET (2006) Expression of UDPglucuronosyltransferase isoform mRNAs during inflammation and infection in mouse liver and kidney. Drug Metab Dispos 34:351-353.

Shah YM, Ma X, Morimura K, Kim I, and Gonzalez FJ (2007) Pregnane X receptor activation ameliorates DSS-induced inflammatory bowel disease via inhibition of NF-kappaB target gene expression. Am J Physiol Gastrointest Liver Physiol 292:G1114-G1122.

Strauss KA, Robinson DL, Vreman HJ, Puffenberger EG, Hart G, and Morton DH (2006) Management of hyperbilirubinemia and prevention of kernicterus in 20 patients with Crigler-Najjar disease. Eur J Pediatr 165:306-319.

Takeuchi O, Hoshino K, Kawai T, Sanjo H, Takada H, Ogawa T, Takeda K, and Akira S (1999) Differential roles of TLR2 and TLR4 in recognition of Gram-negative and Gram-positive bacterial cell wall components. Immunity 11:443-451. 
Tavano R, Franzoso S, Cecchini P, Cartocci E, Oriente F, Aricò B, and Papini E (2009) The membrane expression of Neisseria meningitidis adhesin A (NadA) increases the proimmune effects of MenB OMVs on human macrophages, compared with NadA-OMVs, without further stimulating their proinflammatory activity on circulating monocytes. J Leukoc Biol 86:143-153.

Troutman TD, Bazan JF, and Pasare C (2012) Toll-like receptors, signaling adapters and regulation of the pro-inflammatory response by PI3K. Cell Cycle 11:3559-3567.

van Nood E, Vrieze A, Nieuwdorp M, Fuentes S, Zoetendal EG, de Vos WM, Visser CE, Kuijper EJ, Bartelsman JF, Tijssen JG, et al. (2013) Duodenal infusion of donor feces for recurrent Clostridium difficile. $N$ Engl J Med 368:407-415.

Vanaja SK, Russo AJ, Behl B, Banerjee I, Yankova M, Deshmukh SD, and Rathinam VAK (2016) Bacterial outer membrane vesicles mediate cytosolic localization of LPS and caspase-11 activation. Cell 165:1106-1119.

Venkatesh M, Mukherjee S, Wang H, Li H, Sun K, Benechet AP, Qiu Z, Maher L, Redinbo MR, Phillips RS, et al (2014) Symbiotic bacterial metabolites regulate gastrointestinal barrier function via the xenobiotic sensor PXR and Toll-like receptor 4. Immunity 41:296-310.
Vigsnæs LK, Brynskov J, Steenholdt C, Wilcks A, and Licht TR (2012) Gram-negative bacteria account for main differences between faecal microbiota from patients with ulcerative colitis and healthy controls. Benef Microbes 3:287-297.

Zariri A, Beskers J, van de Waterbeemd B, Hamstra HJ, Bindels TH, van Riet E, van Putten JP, and van der Ley P (2016) Meningococcal outer membrane vesicle composition-dependent activation of the innate immune response. Infect Immun 84:3024-3033.

Zhou X, Xie Y, Qi Q, Cheng X, Liu F, Liao K, Wang G, and Hao H (2013) Disturbance of hepatic and intestinal UDP-glucuronosyltransferase in rats with trinitrobenzene sulfonic acid-induced colitis. Drug Metab Pharmacokinet 28:305-313.

Address correspondence to: Dr. Ru Yan, State Key Laboratory of Quality Research in Chinese Medicine, Institute of Chinese Medical Sciences, University of Macau, Taipa, Macao 999078, China. E-mail: ruyan@umac.mo 